\title{
A REPORT ON STABLE GRAPHS
}

\author{
D. A. HOLTON \\ (Received 21 October 1970) \\ Communicated by J. N. Crossley
}

\section{Introduction}

It is the aim of this paper to introduce a new concept relating various subgroups of the automorphism group of a graph to corresponding subgraphs. Throughout $\mathscr{G}$ will denote a (Michigan) graph on a vertex set $V(|V|=n)$ and $\Gamma(\mathscr{G})=G$ will be the automorphism group of $\mathscr{G}$ considered as a permutation group on $V . E_{n}, C_{n}, D_{n}$ and $S_{n}$ are the identity, cyclic, dihedral, and symmetric groups acting on a set of size $n$, while $S_{p}(q)$ is the permutation group of $p q$ objects which is isomorphic to $S_{p}$ but is $q$-fold in the sense that the objects are permuted $q$ at a time [6]. $H \leqq G$ means that $H$ is a subgroup of $G$. Other group concepts can be found in Wielandt [7]. The graphs $\mathscr{G}_{1} \cup \mathscr{G}_{2}, \mathscr{G}_{1}+\mathscr{G}_{2}, \mathscr{G}_{1} \times \mathscr{G}_{2}$, and $\mathscr{G}_{1}\left[\mathscr{G}_{2}\right]$ along with their corresponding groups are as defined in, for example, Harary [4]. Finally we use $\mathscr{K}_{n}$ for the complete graph on $n$ vertices.

\section{Stable Graphs}

Let $\mathscr{G}_{{ }_{v_{1} v_{2} \ldots v_{k}}}$ be the graph obtained from $\mathscr{G}$ by removing the vertices $v_{1}, v_{2}, \cdots, v_{k}$ and any edges attached to these vertices, from $\mathscr{G}$. As in Wielandt [7] we let $G_{v_{1} v_{2} \ldots v_{k}}$ be the set of permutations of $G$ which keep $v_{1}, v_{2}, \cdots, v_{k}$ individually fixed. Then if for $\mathscr{G}$ there exists a sequences $\mathscr{S}=\left\{v_{1}, v_{2}, \cdots, v_{n}\right\}$ such that $\Gamma\left(\mathscr{G}_{v_{1} v_{2} \ldots v_{k}}\right)=G_{v_{1} v_{2} \ldots v_{k}}$ for all $k=1,2,3, \cdots, n$ we say that $\mathscr{G}$ is stable. Otherwise it is unstable. We refer to the sequence $\mathscr{S}$ as the stabilising sequence of $\mathscr{G}$.

The concept of stability is not vacuous.

Proposition 1. $\mathscr{K}_{n}$ is stable.

Proof. For any $v \in, V\left(\mathscr{K}_{n}\right)_{v}=\mathscr{K}_{n-1}$. Now since $\Gamma\left(\mathscr{K}_{n}\right)=S_{n},\left[\Gamma\left(\mathscr{K}_{n}\right)\right]_{v}=$ $\Gamma\left[\left(\mathscr{K}_{n}\right)_{v}\right]=S_{n-1}$. Then induction shows that any ordering of $V$ is a stability sequence of $\mathscr{K}_{n}$. 
The following four propositions are readily established. stable.

Proposition 2. If $\mathscr{G}_{v}$ is stable for some $v \in V$, and $\Gamma\left(\mathscr{G}_{v}\right)=G_{v}$ then $\mathscr{G}$ is

Proposition 3. If $\mathscr{G}$ is stable then there exists a $v \in V$ such that $\mathscr{G}_{v}$ is stable.

PRoposition 4. If $\tilde{G}$ is the complement of $\mathscr{G}$ on $V$ we have $\mathscr{G}$ is stable if and only if $\tilde{\mathscr{G}}$ is stable.

Proposition 5. If $\mathscr{G}_{v}$ is unstable for all $v \in V$ then $\mathscr{G}$ is unstable.

\section{A class of unstable graphs}

Here we show that, as was to be expected, the attribute of stability depends to a large extent, but not completely, on the automorphism group of the graph.

Lemma 1. If $G \leqq D_{n}$ then $G_{v} \leqq D_{n-1}$ for all $v \in V$.

Proof. Now $G_{v} \leqq\left(D_{n}\right)_{v}$ so it is enough to show that $\left(D_{n}\right)_{v} \leqq D_{n-1}$.

By the orbit-stabiliser relation, and bearing in mind that $D_{n}$ is transitive we have

$$
\left|\left(D_{n}\right)_{v}\right|=\frac{\left|D_{n}\right|}{\left|D_{n}\right|}=\frac{2 n}{n}=2 .
$$

The elements of order two in $D_{n}$ are the product of $(n-1) / 22$-cycles if $n$ is odd and the product or $n / 2$ or $(n-2) / 22$-cycles if $n$ is even and hence an element of order two in $D_{n}$ which fixes one vertex belongs to $D_{n-1}$.

Clearly then $\left(D_{n}\right)_{v} \leqq D_{n-1}$ since $D_{n-1}=\langle(123 \cdots n-1),(1 n-1)(2 n-2) \cdots\rangle$ and we would have $\left(D_{n}\right)_{v}=\langle(1 n-1)(2 n-2) \cdots\rangle$ where the members of $V$ have been reordered if necessary.

We can now prove

THEOREM 1. If $\mathscr{G}$ is a graph on $n$ vertices $V$ with $\Gamma(\mathscr{G})=G \leqq D_{n}$ and if $n \geqq 5$ then $\mathscr{G}$ is unstable.

Proof. If $n=5$ then $G$ could be $D_{5}, C_{5}, S_{2}(2)+E_{1}$, or $E_{5}$. However $C_{5}$ and $E_{5}$ do not appear as automorphism groups for any graph on five vertices.

(i) $D_{5}$. The only graph whose automorphism group is $D_{5}$ is shown in Figure 1(a). Now for any vertex $v_{1}$ of $(a) \Gamma\left(\mathscr{G}_{v_{1}}\right)=\left(D_{5}\right)_{v_{1}}$, but for another vertex $v_{2}$ we find that $\left(D_{5}\right)_{v_{1} v_{2}}=E_{3}$ and $\Gamma\left(\mathscr{G}_{v_{1} v_{2}}\right) \neq E_{3}$. Hence the pentagon of Figure $1(a)$ is unstable.

(ii) $S_{2}(2)+E_{1}$. The graphs $(b)$ to $(f)$ inclusive in Figure 1 all have $S_{2}(2)+E_{1}$ as their automorphism groups.

(b), (c). The graphs (b) and (c) are complements and so by Proposition 4 it is sufficient to discuss $(b)$. 


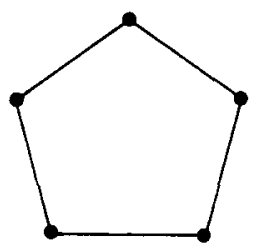

(a)

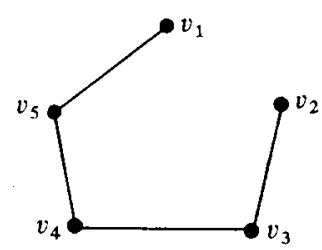

(d)

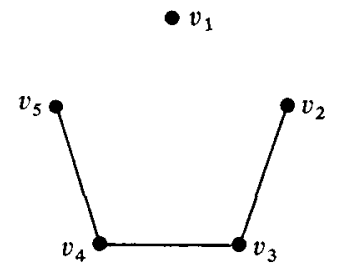

(b)

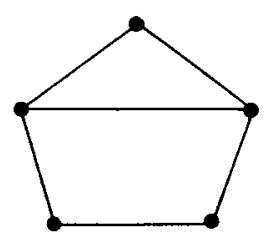

(e)

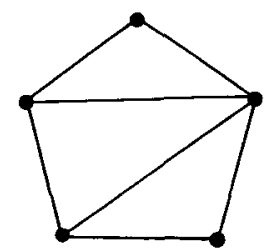

(c)

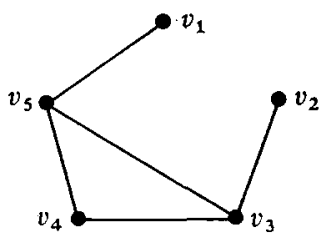

(f)

Figure 1.

Clearly $\Gamma\left(\mathscr{G}_{v_{1}}\right)=S_{2}(2)=\left(S_{2}(2)+E_{1}\right)_{v_{1}}$. But $\left(S_{2}(2)+E_{1}\right)_{v_{1} v_{i}}=E_{3} \quad$ for $i=2,3,4,5$ and $\Gamma\left(\mathscr{G}_{v_{1} v_{i}}\right) \neq E_{3}$. Hence $(b)$ (and $\left.(c)\right)$ is unstable.

$(d),(e)$. Again these graphs are complements so we discuss $(d)$ only.

Here $\Gamma(\mathscr{G})=S_{2}(2)+E_{1}=\left\{e,\left(v_{1} v_{2}\right)\left(v_{3} v_{5}\right)\right\}$ and hence $\left(S_{2}(2)+E_{1}\right)_{v_{4}}=$ $S_{2}(2)$. But $\Gamma\left(\mathscr{G}_{v_{4}}\right)=S_{2}\left[S_{2}\right] \neq S_{2}(2)$. Further for $i \neq 4\left(S_{2}(2)+E_{1}\right)_{v_{i}}=E_{4}$ but $\Gamma\left(\mathscr{G}_{v_{i}}\right) \neq E_{4^{*}}$. Thus $(d)$ is not stable.

$(f)$. In this case $\Gamma(\mathscr{G})=\left\{e,\left(v_{1} v_{2}\right)\left(v_{3} v_{5}\right)\right\}$. For $i=1,2 \Gamma\left(\mathscr{G}_{v_{i}}\right)=\left\{e,\left(v_{4} v_{5}\right\}\right)$ which is not of the form $\left(S_{2}(2)+E_{1}\right)_{v_{i}}$. With $j=3,5 \Gamma\left(\mathscr{G}_{v_{j}}\right)=\left\{e,\left(v_{i} v_{4}\right)\right\} i=1,2$, and again this is not a subgroup of $S_{2}(2)+E_{1}$. However $\Gamma\left(\mathscr{G}_{v_{4}}\right)=\left(S_{2}(2)+E_{1}\right)_{v_{4}}$, but then $\left(S_{2}(2)+E_{1}\right)_{v_{4} v_{l}}=E_{3}(l=1,2,3,5)$ and $\Gamma\left(\mathscr{G}_{v_{4} v_{l}}\right) \neq E_{3}$.

Thus the theorem is true for $n=5$.

We now assume that the theorem is true for $n-1 \geqq 5$. Let $|V|=n$ and $V^{\prime}=\left\{v \in V: G_{v}=\Gamma\left(\mathscr{G}_{v}\right)\right\}$. We need not worry about the vertices of $V-V^{\prime}$ since in trying to find a first member of the stabilising sequence for $\mathscr{G}$ these vertices must be ignored.

But $G_{v} \leqq D_{n-1}$ by Lemma 1 and so by the induction hypotheses $\mathscr{G}_{v}$ is unstable. This is true for all $v \in V-V^{\prime}$ and so $\mathscr{G}$ is unstable.

So we can see that the automorphism group of the graph plays a large role in the determination of stability. However it is not the sole factor involved.

Consider the graphs of Figure 2. For both graphs the automorphism group is $S_{2}(2)+S_{2}$. But in fact $(a)$ is stable while $(b)$ is not. For $(a) \Gamma(G)=\left\{e,\left(v_{2} v_{5}\right)\right.$, $\left.\left(v_{1} v_{4}\right)\left(v_{3} v_{6}\right),\left(v_{1} v_{4}\right)\left(v_{2} v_{5}\right)\left(v_{3} v_{6}\right)\right\}$ and $v_{1}, v_{3}, v_{4}, v_{6}, v_{2}, v_{5}$ is a stabilising sequence.

For $(b) \Gamma(\mathscr{G})=\left\{e,\left(v_{5} v_{6}\right),\left(v_{1} v_{4}\right)\left(v_{2} v_{3}\right),\left(v_{1} v_{4}\right)\left(v_{2} v_{3}\right)\left(v_{5} v_{6}\right)\right\}$. Trouble arises in the stabilising sequence when one tries to remove one of the points $v_{1}, v_{2}, v_{3}$, or 

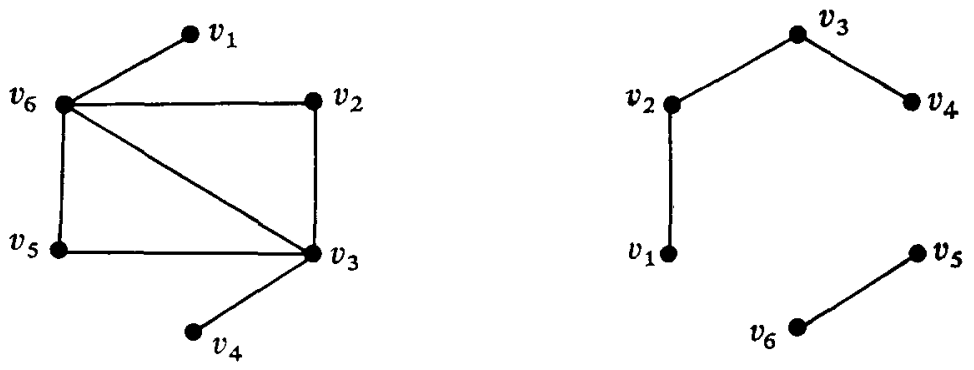

Figure 2.

$v_{4}$ from the graph. (As it happens $\mathscr{G}_{v_{5} v_{6}}$ is unstable and so the unstability of $\mathscr{G}$ is guaranteed by Theorem 5.)

At this stage it can only be said that most likely connectedness plays a part in stability. One sees that for $(a) \mathscr{G}$ and $\tilde{\mathscr{G}}$ are connected while for $(b) \tilde{\mathscr{G}}$ is connected while $\mathscr{G}$ obviously is not. We conjecture then that for graphs $\mathscr{G}$ and $\mathscr{K}$, if $\mathscr{G}, \tilde{G}, \mathscr{K}$ and $\tilde{\mathscr{K}}$ are all connected and if $\Gamma(\mathscr{G})=\Gamma(\mathscr{K})$ then $\mathscr{G}$ is stable if $\mathscr{K}$ is. Similar conjectures arise for unstability and also in the cases where $\mathscr{G}$ and $\mathscr{K}$ are connected and $\tilde{\mathscr{G}}$ and $\tilde{\mathscr{K}}$ are not.

\section{The union of stable graphs}

Here we confine our attention to stable graphs and show that the union of $n$ graphs $\left(\bigcup_{i=1}^{n} \mathscr{G}_{i}\right)$ is stable if and only if each of the graphs $\mathscr{G}_{i}$ is. We first note the well known Theorems 2 and 3, and prove Theorem 4.

THEOREM 2. $\Gamma\left(\mathscr{G}_{1} \cup \mathscr{G}_{2}\right)=\Gamma\left(\mathscr{G}_{1}\right)+\Gamma\left(\mathscr{G}_{2}\right)$ if and only if no component of $\mathscr{G}_{1}$ is isomorphic to a component of $\mathscr{G}_{2}$.

THEOREM 3. Frucht [2]: If $\mathscr{G}_{1}=\mathscr{G}_{2}$ and $\mathscr{G}_{1}$ is connected then $\Gamma\left(\mathscr{G}_{1} \cup \mathscr{G}_{2}\right)$ $=S_{2}\left[\Gamma\left(\mathscr{G}_{1}\right)\right]$.

The extensions of the above two theorems to the union of $n$ graphs are clear.

TheOREM $4^{1}$. For all positive integers $n, S_{n}[G]_{v}=S_{n-1}[G]+G_{v}$.

Proof. Let $S_{n}$ act on $\Omega=\{1,2,3, \cdots, n\}$ and $G$ act on

$$
\begin{aligned}
& \Sigma=\{1,2,3, \cdots, m\} . \text { Form } \Omega \times \Sigma \text { and let } \\
& \Sigma_{i}=\{(i, j): i \in \Omega \text { is fixed and } j \in \Sigma\} \text {. Clearly } \Sigma_{i} \text { is just a copy of } \Sigma .
\end{aligned}
$$

Now $\Sigma_{i}^{g}=\Sigma_{i^{\prime}}$ for all $g \in S_{n}[G]$ and for some $i^{\prime} \in \Omega$. Further if $v=(\alpha, \beta)$ and $h \in S_{n}[G]_{v}$ then $\Sigma_{\alpha}^{h}=\Sigma \alpha$. But $S_{n}[G]_{v}$ acts on this set as $G_{v}$ (if we identify $\beta$ with $(\alpha, \beta)$ here) and as $S_{n-1}[G]$ on $\Sigma^{\prime}=(\Omega \times \Sigma)-\Sigma_{\alpha}$.

${ }^{1}$ The proof here was suggested by Prof. W. Jonsson of McGill University. 
Then since $\Omega \times \Sigma=\Sigma^{\prime} \cup \Sigma_{\alpha}$ and $\Sigma^{\prime} \cap \Sigma_{\alpha}=\phi$ it can be seen that $S_{n}[G]_{v}=$ $S_{n-1}[G]+G_{v}$.

We can now prove

Theorem 5. $\bigcup_{i=1}^{n} \mathscr{G}_{i}$ is stable if and only if each $\mathscr{G}_{i}$ is stable.

Proof. (a) We first assume that all the $\mathscr{G}_{i}$ are connected and ordered so that for $j<k,\left|V_{j}\right| \leqq\left|V_{k}\right| .(\Leftrightarrow)$ If $\mathscr{G}_{i}$ has stabilising sequence $\left\{v_{i 1}, v_{i 2}, \cdots, v_{i m_{i}}\right\}$ then $\left.v_{11}, v_{12}, \cdots, v_{1 m_{1}}, v_{21}, \cdots, v_{2 m_{2}}, \cdots, v_{n 1}, \cdots, v_{n m_{n}}\right\}$ is a stabilising sequence of $\bigcup_{i=1}^{n} \mathscr{G}_{i}$. For if $\mathscr{K}=\bigcup_{i=1}^{n} \mathscr{G}_{i}$ then $\mathscr{K}_{v_{11}}=\left(\mathscr{G}_{1}\right)_{v_{11}} \cup\left(\bigcup_{i=2}^{n} \mathscr{G}_{i}\right)$ and by the stability of $\mathscr{G}_{1}$ and using theorems 2,3 and 4 we have $\Gamma\left(\mathscr{K}_{v_{11}}\right)=\Gamma(\mathscr{K})_{v_{11}}$. Induction then produces the required result.

$(\Rightarrow)$ Let $\left\{v_{1}, v_{2}, \cdots, v_{m}\right\}$ be a stabilising sequence of $\mathscr{K}=\bigcup_{i=1}^{n} \mathscr{G}_{i}$. Assume $v_{1} \in \mathscr{G}_{1}$ (reorder if necessary). Since $\mathscr{K}$ is stable $\Gamma\left(\mathscr{K}_{v_{1}}\right)=\Gamma(\mathscr{K})_{v_{1}}$.

Now

$$
\begin{aligned}
\Gamma\left(\mathscr{K}_{v_{1}}\right) & =\Gamma\left(\left[\mathscr{G}_{1}\right]_{v_{1}} \cup \bigcup_{i=2}^{n} \mathscr{G}_{i}\right) \\
& =\Gamma\left(\left[\mathscr{G}_{1}\right]_{v_{1}}\right)+\Gamma\left(\bigcup_{i=2}^{n} \mathscr{G}_{i}\right) \quad \text { provided }
\end{aligned}
$$

$\left[\mathscr{G}_{1}\right]_{v_{1}} \neq \mathscr{G}_{i_{0}}$ for some $i_{0}=2,3, \cdots, n$, when a wreath product would occur

$$
\text { Further } \Gamma(\mathscr{K})=\left\{\begin{array}{l}
S_{r}\left[\mathscr{G}_{1}\right]+\Gamma\left(\bigcup \mathscr{G}_{i}\right) \\
\Gamma\left(\mathscr{G}_{1}\right)+\Gamma\left(\bigcup_{i=2}^{n} \mathscr{G}_{i}\right)
\end{array}\right.
$$

depending on whether $\mathscr{K}$ contains $r$ connected components identical with $\mathscr{G}_{1}$ or not. In the first case the $\bigcup \mathscr{G}_{i}$ is over all $\mathscr{G}_{i} \neq \mathscr{G}_{1}$.

Then

$$
\begin{aligned}
\Gamma(\mathscr{K})_{v_{1}} & =\left\{\begin{array}{l}
\left(S_{r}\left[\mathscr{G}_{1}\right]+\Gamma\left(\bigcup \mathscr{G}_{i}\right)\right)_{v_{1}} \\
\left(\Gamma\left(\mathscr{G}_{1}\right)+\Gamma\left(\bigcup_{i=2}^{n} \mathscr{G}_{i}\right)\right)_{v_{1}}
\end{array}\right. \\
& =\left\{\begin{array}{l}
\Gamma\left(\mathscr{G}_{1}\right)_{v_{1}}+S_{r-1}\left[\mathscr{G}_{1}\right]+\Gamma\left(\bigcup \mathscr{G}_{i}\right) \\
\Gamma\left(\mathscr{G}_{1}\right)_{v_{1}}+\Gamma\left(\bigcup_{i=2}^{n} \mathscr{G}_{i}\right)
\end{array}\right. \\
& =\Gamma\left(\mathscr{G}_{1}\right)_{v_{1}}+\Gamma\left(\bigcup_{i=2}^{n} \mathscr{G}_{i}\right) .
\end{aligned}
$$

And since $\mathscr{K}$ is stable we have $\left.\Gamma\left(\mathscr{G}_{1}\right)_{v_{1}}=\Gamma\left(\left[\mathscr{G}_{1}\right]\right)_{v_{1}}\right)$ provided $\left[\mathscr{G}_{1}\right]_{v_{1}} \neq \mathscr{G}_{i_{0}}$.

If $\left[\mathscr{G}_{1}\right]_{v_{1}}=\mathscr{G}_{l_{0}}$ then 


$$
\Gamma\left(\mathscr{K}_{v_{1}}\right)=S_{u}\left[\Gamma\left(\left[\mathscr{G}_{1}\right]_{v_{1}}\right)\right]+\Gamma\left(\bigcup_{i \in A} \mathscr{G}_{i}\right)
$$

and

$$
\begin{aligned}
\Gamma(\mathscr{K})_{v_{1}} & =\left(S_{u-1}\left[\Gamma\left(\mathscr{G}_{i_{0}}\right)\right]+\Gamma\left(\bigcup_{i \in B} \mathscr{G}_{i}\right)\right)_{v_{1}} \\
& =S_{u-1}\left[\Gamma\left(\mathscr{G}_{i_{0}}\right)\right]+\Gamma\left(\mathscr{G}_{1}\right)_{v_{1}}+\Gamma\left(\bigcup_{i \in A} \mathscr{G}_{i}\right) .
\end{aligned}
$$

Here the union over A excludes only the $u$ copies of $\mathscr{G}_{i_{0}}$ and that over $B$ excludes the $u-1$ copies of $\mathscr{G}_{i_{0}}$.

But $S_{u-1}\left[\Gamma\left(\left[\mathscr{G}_{1}\right]_{v_{1}}\right]=S_{u-1}\left[\Gamma\left(\mathscr{G}_{i_{0}}\right)\right]\right.$. However $S_{u}\left[\Gamma\left(\left[\mathscr{G}_{1}\right]_{v_{1}}\right)\right] \neq S_{u-1}\left[\Gamma\left(\left[\mathscr{G}_{1}\right]_{v_{1}}\right)\right]$ $+\Gamma\left(\mathscr{G}_{1}\right)_{v_{1}}$ and so $\Gamma\left(\mathscr{K}_{v_{1}}\right) \neq \Gamma(\mathscr{K})_{v_{1}}$ which contradicts $v_{1}$ 's position in the stabilising sequence of $\mathscr{K}$. Hence $\left[\mathscr{G}_{1}\right]_{v_{1}} \neq \mathscr{G}_{i_{0}}$.

The proof is again completed by induction. We note that a stabilising sequence for $\mathscr{G}_{i}$, can be obtained from that of $\mathscr{K}$ by taking the vertices of $\mathscr{G}_{i}$, in the order that they appear in the stabilising sequence of $\mathscr{K}$.

(b) If $\mathscr{G}_{i}$ not connected $\mathscr{G}_{i}=\bigcup_{j=1}^{s} \mathscr{F}_{i j}$ where the $\mathscr{F}_{i j}$-are connected.

By part $(a) \mathscr{G}_{i}$ is stable if and only if each $\mathscr{F}_{i j}$ is stable. But $\mathscr{K}=\bigcup_{i=1}^{n} \mathscr{G}_{i}=$ $\bigcup_{i=1}^{n} \bigcup_{j=1}^{s} \mathscr{F}_{i j}$. So if $\mathscr{K}$ is stable then each $\mathscr{F}_{i j}$ is by part $(a)$ and so each $\mathscr{G}_{i}$ is. On the other hand when each $\mathscr{G}_{i}$ is stable so is each $\mathscr{F}_{i j}$ and by $(a)$ so is $\mathscr{K}$.

Corollary. $\mathscr{G}_{1}+\mathscr{G}_{2}+\cdots+\mathscr{G}_{n}$ is stable if and only if each $\mathscr{G}_{i}$ is stable.

Proof. Now the complement of $\mathscr{G}_{1}+\mathscr{G}_{2}+\cdots+\mathscr{G}_{n}=\bigcup_{i=1}^{n} \tilde{\mathscr{G}}_{i}$. So by the theorem the complement of $\mathscr{G}_{1}+\mathscr{G}_{2}+\cdots \mathscr{G}_{n}$ is stable if and only if each $\tilde{\mathscr{G}}_{i}$ is.

The corollary then follows by application of Proposition 4.

One would hope that the result of Theorem 6 and its corollary could be extended to the Cartesian product of two (or more) graphs. However consider the following counter example.

The Cartesian product [3], $\mathscr{G}_{1} \times \mathscr{G}_{2}$, of $\mathscr{G}_{1}$ and $\mathscr{G}_{2}$ is as shown in Figure 3.
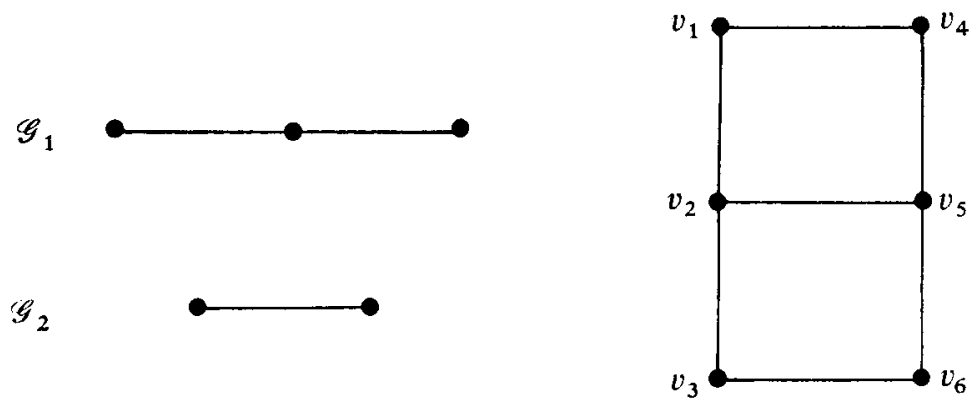

$\mathscr{G}_{1} \times \mathscr{G}_{2}$

$\mathscr{G}_{2}$

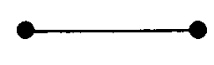

Figure 3.

$\mathscr{G}_{1}$ and $\mathscr{G}_{2}$ are quickly seen to be stable. But $\Gamma\left(\mathscr{G}_{1} \times \mathscr{G}_{2}\right)<D_{6}$ and so by Theorem $1, \mathscr{G}_{1} \times \mathscr{G}_{2}$ is unstable. 


\section{Special examples}

We consider below the stability of three well known graphs.

(a) Petersen's Graph is Unstable.

The automorphism group of the Petersen graph, $P$, Figure 4, is well known [1]. It has degree 10 , order 120 , is isomorphic to $S_{5}$ and $\Gamma(P)=\langle(1429)(3765)$ $(810),(13)(46)(58)>$.

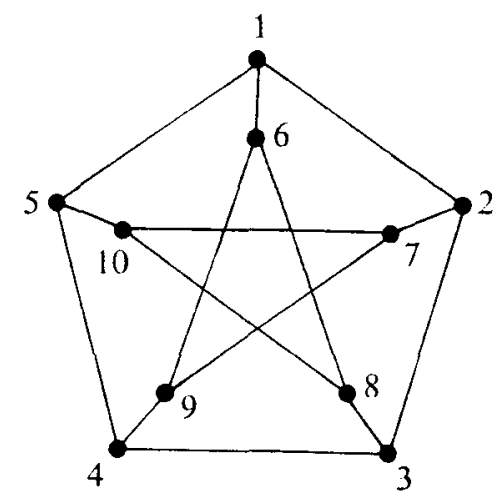

Figure 4.

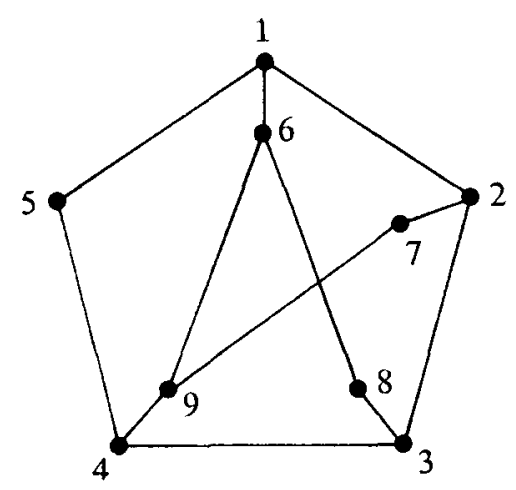

Figure 5.

Now $P_{v_{1}}=P_{v_{2}}$ for all $v_{1}, v_{2} \in V$ the vertex set of $P$, and so to test for stability we remove an arbitrary point, 10 say. Figure 5 shows $P_{10}$.

After some work we have $\Gamma\left(P_{10}\right)=\Gamma(P)_{10}=\langle(169432)(587),(14)(23)$ (69)(78) $\rangle$, and $\left|\Gamma\left(P_{10}\right)\right|=12$.

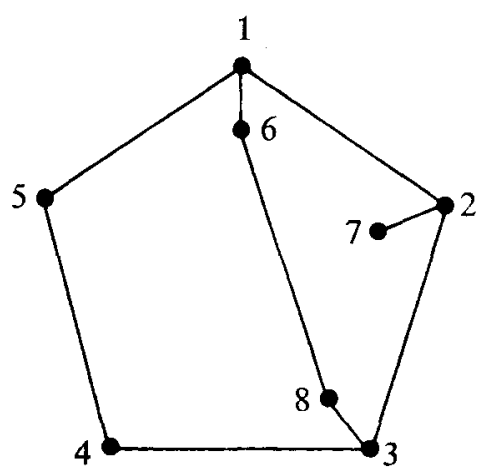

Figure 6.

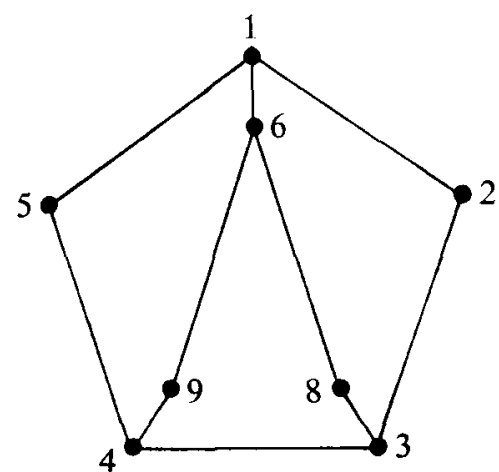

Figure 7.

In $P_{10}$ there are six vertices with degree 3 and three with degree 2 . Removing any one of the former set of vertices from $P_{10}$ gives a graph isomorphic to $P_{10,9}$ (Figure 6). On the other hand $P_{10.7}$ is isomorphic to any graph formed by removing a vertex of degree 2 from $P_{10}$ (Figure 7). 
Now 9 is in an orbit of $\Gamma\left(P_{10}\right)$ of length 6 and 7 is in an orbit of $\Gamma\left(P_{10}\right)$ of length 3 and so by the orbit stabiliser relation we have

and

$$
\left|\Gamma\left(P_{10}\right)_{9}\right|=\frac{\left|\Gamma\left(P_{10}\right)\right|}{\left|9^{\left(P_{10}\right)}\right|}=\frac{12}{6}=2,
$$

$$
\left|\Gamma\left(P_{10}\right)_{7}\right|=\frac{\left|\Gamma\left(P_{10}\right)\right|}{\left|7\left(P_{10}\right)\right|}=\frac{12}{3}=4 .
$$

However it can be quickly seen that

(56) (48), (13) (45) (68), (13) (58) (46) $\in \Gamma\left(P_{10,9}\right)$ and (25) (34) (89), (14) (36) (29), (13) (46) (58), (16) (34) (29) (58), (16) (28) (59), (1364) (2895), (1463) (2598) $\epsilon$ $\Gamma\left(P_{10,7}\right)$. Hence $\Gamma\left(P_{10,9}\right) \neq \Gamma\left(P_{10}\right)_{9}$ and $\Gamma\left(P_{10,7}\right) \neq \Gamma\left(P_{10}\right)_{7}$, and so $P$ cannot be stable.

(b) Desargues' graph is unstable.

As a corollary to (a) then, we see that Desargues' graph is also unstable, since this graph is the complement of $P$ [5].

(c) The Pappus Graph is Stable

The complement of the Pappus graph (see for example [5]) is shown in Figure 8.
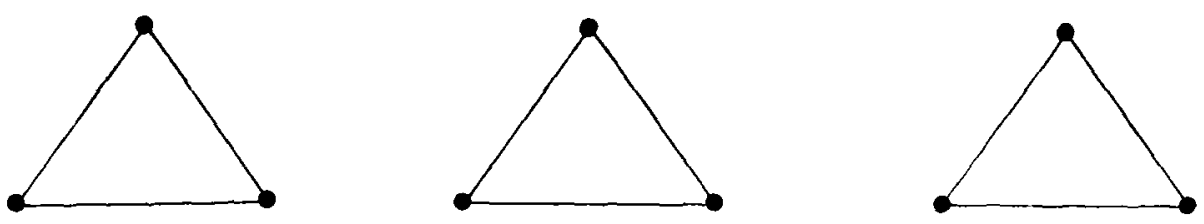

Figure 8.

This is clearly the sum of three graphs $K_{3}$. But $K_{3}$ is stable (Proposition 1) and hence $K_{3} \cup K_{3} \cup K_{3}$ is stable (Theorem 5). Hence by Proposition 4 the Pappus graph is stable.

\section{Stability of graphs for $2 \leqq|V| \leqq 6$}

Since either a graph or its complement must be connected we consider only connected graphs. A list of these for $2 \leqq|V| \leqq 6$ will be found in Appendix 3 of [6]. For a given $|V|$ we number the graphs of that list in the order they occur. Below we note only the unstable graphs.

(i) $|V|=2,3$, all stable

(ii) $|V|=4$, only graph 1 unstable

(iii) $|V|=5$, graphs $2,4,8,12$, and 16 are unstable 
(iv) $|V|=6, \quad$ graphs $1,4,9,12,14,18,19,24,30,31,32,36,37,44,45$, $46,53,54,55,59,69,70,71,74,77,79,85,88,91,93,100$, 102, and 106 are unstable. (N. B. Graph 20 for $|V|=6$ should be as in Figure 9).

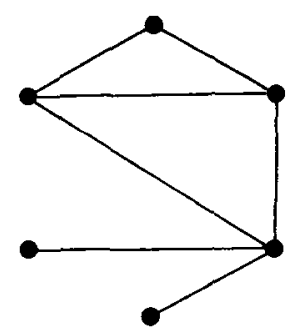

Figure 9.

\section{References}

[1] R. Frucht, 'Die Gruppe des Petersenchen Graphen und der Kantensysteme der regulären Polyeder', Comm. Math. Helv, 9 (1937), 217-223.

[2] R. Frucht, 'On the groups of repeated graphs', Bull. Amer. Math. Soc. 55 (1949), 418-420.

[3] F. Harary, 'On the group of the composition of two graphs', Duke Math. Journal 26 (1959), 29-34.

[4] F. Harary, Graph Theory (Addison-Wesley (1969)).

[5] I. Kagno, 'Desargues' and Pappus 'graphs and their groups', Amer. J. Math. 69 (1947), 859-862.

[6] G. Uhlenbeck and L. Ford, Theory of Linear Equations in Studies in Statistical Mechanics, Vol. 1 Ed. Boer and G. Uhlenbeck, (North Holland Publishing Co. (1962)).

[7] H. Wielandt, Finite Permutation Groups (Academic Press (1964)).

Department of Mathematics

University of Melbourne

Parkville, Vic 3052

Australia 